\title{
EL PODER DE LA DECEPCIÓN: UN ANÁLISIS DEL EFECTO BOCA-OREJA EN LAS PELÍCULAS
}

\author{
JOSÉ IGNACIO AZUELA FLORES \\ Universidad Autónoma de Tamaulipas \\ Facultad de Comercio y Administración de Tampico, México \\ iazuelaf@uat.edu.mx \\ NADIA HUITZILIN JIMENEZ TORRES \\ Universidad de Burgos, España \\ nhjimenez@ubu.es
}

\section{Resumen}

Este artículo analiza los efectos de las experiencias "emocionantes" y "decepcionantes" en el cine, sobre la cantidad de comunicación boca-oreja que generan los individuos en las redes sociales. Para ello, se realizó un diseño pre-experimental de grupo único con prueba previa y posterior. Para tal efecto se realizó una Prueba t para muestras relacionadas que permitió identificar los efectos específicos de una película emocionante o decepcionante sobre el boca-oreja. Los resultados muestran diferencias significativas entre ambos tipos de experiencia; películas emocionantes y películas decepcionantes $(p=.000)$. Así pues, la diferencia media entre ambas condiciones es significativamente diferente. De tal modo que cuando las experiencias fueron positivas (películas emocionantes), los individuos tienden a hablar menos de la película en las redes sociales (RR.SS.) Mientras que cuando se enfrentan a una experiencia negativa (película decepcionante), los participantes tienden a hablar más.

Palabras Clave: Experimentación, Boca-oreja virtual, Boca-oreja electrónico, Redes Sociales, Cine.

JEL: M31, Z11.

\begin{abstract}
This paper analyses "exciting" and "disappointing" experiences to movies and their effects on the volume of word-of-mouth communication on social networks. To achieve this objective, an experiment was designed with 281 participants. Our results show that, when the experiences are positive (exciting movies), the consumers tend to generate less WOM about that movie on social networks than when the experiences are negative (disappointing movies).
\end{abstract}

Keywords: Experiment, virtual word-of-mouth, eWOM, Social Networks, Movies Theater.

JEL: M31, Z11

\section{INTRODUCCIÓN}

En el cine, como en todos los productos de experiencia, es difícil determinar a priori la calidad de una película (Reinstein y Snyder, 2005). Lo anterior, confiere un alto grado de riesgo e incertidumbre al consumo de cine. Para evitar el riesgo asociado al consumo, los individuos tienden a acudir a toda aquella información disponible que les permita determinar la calidad de una película. 
La comunicación boca-oreja', y en general toda la información disponible previa al consumo, puede cumplir esa función. Sin embargo, frente a la información emitida o publicitada por las empresas, la información boca-oreja es recibida con mayor credibilidad entre los consumidores ${ }^{2}$.

De hecho, se ha señalado que, el boca-oreja es el tipo de comunicación informal privada que más influye en el comportamiento del consumidor (Bansal y Voyer, 2000; Casaló et al. 2008). De tal forma que, la confianza que un consumidor deposite en una empresa puede estar influida por lo que opinen terceras personas (Granovetter, 1973). Esto se debe, principalmente, a la dificutad para evaluar la calidad de un servicio en ausencia de experiencias previas (Kyung et al. 2004; Yongseog, 2005). Así pues, la confianza que los individuos otorguen a las opiniones de otros será mayor, cuando menores sean sus experiencias de consumo (Gilly et. al. 1998; Bansal y Voyer, 2000).

La naturaleza del boca-oreja, en la que los individuos no persiguen los intereses económicos de las empresas, conduce a flujos de información tanto positivos como negativos. En otras palabras, los individuos comparten entre sí opiniones positivas y negativas sobre los productos/ servicios. Esta dualidad del boca-oreja muestra efectos asimétricos sobre el comportamiento y actitudes de los consumidores. De manera que, frente al boca-oreja positivo, es mayor la influencia del boca-oreja negativo sobre el comportamiento (Katz y Lazarsfeld, 1955; Chakravarty et al. 2010), y la actitud de los consumidores (Engel et al. 1969).

Asimismo, se ha demostrado que el efecto del boca oreja negativo difiere en función de la

1 En adelante WOM por sus siglas en ingles Word-of-Mouth

2 Se ha demostrado que la comunicación boca-oreja ejerce mayor influencia no sólo frente a la información proporcionada por las empresas sino que también frente a aquella información proporcionada por agentes, en principio, neutrales y sin conflicto de intereses como es el caso de las revistas del consumidor (Herr et al. 1991). categoría de producto. De tal modo que, frente a los productos hedónicos (ej. la música y el cine), el peso de las opiniones negativas crece cuando se trata de productos utilitarios (ej. bienes durables) (Sen y Lerman, 2007).

Naturalmente, tanto empresas como practicantes del marketing están interesados en que la comunicación transmitida entre consumidores sea cuantiosa y positiva. No obstante, el sentido y la cantidad de la comunicación boca-oreja están fuera de su alcance. Sin embargo, y aunque no sea conveniente manipular la información entre consumidores, sí es posible fomentarla. Así pues, la evidencia empírica señala que la comunicación boca-oreja positiva se estimula a través de experiencias de consumo satisfactorias. Mientras que el boca-oreja negativo es resultado de experiencias de consumo insatisfactorias (Richins, 1983).

Como se ha señalado, es posible identificar qué propicia a cada tipo de boca-oreja (positivo y negativo). Además, se sabe que es mayor el impacto del boca-oreja negativo e incluso que éste es aún mayor cuando se trata de bienes utilitarios. Sin embargo, la discusión sobre los efectos del e-WOM (por sus siglas en inglés electronic wordof-mouth/mouse), aún sigue abierta (Yang et al. 2012), y la proliferación de los contenidos generados por los usuarios en diferentes foros como blogs, wikis, mundos virtuales, redes sociales, podcasts, entre otros, confieren al e-WOM un amplio abanico de posibilidades de estudio.

En la industria cinematográfica, donde el ciclo de vida de sus productos es muy corto y donde el fin de semana de estreno es determinante en la carrera comercial de una película (Jedidi et al. 1998), el conocer la cantidad de boca-oreja que generan los distintos tipos de experiencias (satisfactorias e insatisfactorias) supone una herramienta importante para los ejecutivos de la industria. Por tanto, el presente trabajo se centra en medir la cantidad de comunicación boca-oreja que generan tanto las experiencias emocionantes como 
las decepcionantes en el cine. En concreto, nos centramos en la comunicación suscitada en las redes sociales pues, y frente al boca oreja offline, el alcance es mayor y los costes asociados a la transmisión de boca-oreja son inferiores.

Para lograr el anterior objetivo, se realizó un diseño pre-experimental de un grupo único con prueba previa y posterior, en donde los participantes fueron sometidos a dos escenarios. En el primero de ellos, se les situaba en un contexto en el cual habían visionado una película que, en su opinión, fue "emocionante". Mientras que en el segundo, se les instaba a pensar en un escenario donde habían visto una película "decepcionante". Posteriormente, se midió el efecto e-WOM que tenían dichas experiencias.

El resto del trabajo se organiza de la siguiente forma; a continuación se señala la metodología empleada. A renglón seguido, se describe la selección de los participantes, el diseño y las medidas del experimento. Por último, se ofrecen los principales resultados alcanzados y se exponen las principales conclusiones y líneas futuras de investigación.

\section{MATERIALES Y MÉTODO}

Como ya se mencionó, el objetivo de este trabajo es medir la cantidad de comunicación bocaoreja que generan tanto las experiencias emocionantes, como las decepcionantes en el cine.

Teniendo en cuenta que, en la actualidad, los consumidores utilizan las RR.SS. de forma habitual para obtener información sobre productos y servicios (Tran et al. 2012; Arenas-Gaitán et al. 2013), y que además, frente al boca oreja offline, los costes asociados a la transmisión de información boca-oreja virtual son inferiores. En la presente investigación, nos centramos en la comunicación suscitada entre consumidores en las redes sociales.
En congruencia con investigaciones previas (véase por ejemplo; Chung y Tsai, 2009), para lograr el anterior objetivo, se realizó un diseño pre-experimental de un grupo único con prueba previa y posterior, en donde los participantes fueron sometidos a dos escenarios; 1) el de haber visionado una película que, en su opinión, fue "emocionante", y 2) un escenario donde habían visto una película "decepcionante".

En el primer escenario, se les situaba en un contexto en el cual habían visionado una película que, en su opinión, fue "emocionante" (Recuerda la última vez que fuiste al cine y la película que viste fue emocionante). A continuación, se identificó la intención de comunicar la experiencia dentro de las RR.SS. (sí hablaría de la película, no hablaría de la película) y, finalmente, a través de una escala de razón, se registró la cantidad de personas con las que hablarían de la película.

El mismo procedimiento fue empleado para registrar la comunicación boca-oreja que, las experiencias decepcionantes suscitaban en las RR.SS. A los participantes, se les instaba a pensar en un escenario donde habían visto una película "decepcionante" (Ahora recuerda la última vez que fuiste al cine y la película que viste fue decepcionante). Posteriormente, se registró la cantidad de personas con las que hablarían de la película

Posteriormente, se realizó una Prueba t para muestras relacionadas que nos permitió identifcar específicamente los efectos de la experiencia de ver una película emocionante o decepcionante sobre el boca-oreja en RR.SS.

\section{PARTICIPANTES}

Los participantes fueron hombres y mujeres de entre 18 y 30 años ${ }^{3}$ de edad. Todos ellos seleccionados bajo un mismo criterio: ser usuarios de, al menos, una RR.SS. Bajo estas circunstancias, la selección se realizó a través de un muestreo por conveniencia interceptando a los participantes 
en espacios públicos de Tampico, Tamaulipas (México) donde se concentran con mayor frecuencia personas de este segmento (jóvenes de entre 18 y 30 años usuarios de RR.SS.) La muestra final constó de 281 individuos de los cuales el 53.3\% fueron mujeres, y la edad promedio se situó en torno a los 23 años (Desviación estándar = 3.03). Aunque la muestra pueda ser cuestionada, hay que recordar que la finalidad de este trabajo no es la de ofrecer resultados definitivos, sino exploratorios que ayuden a comprender el efecto boca-oreja en las redes sociales virtuales mediante una técnica pre-experimental. Por otra parte, se ha demostrado que a través de muestras homogéneas (como es el caso de la muestra empleada en este trabajo), se obtienen predicciones teóricas de mayor precisión que si se utilizasen muestras heterogéneas (Calder et al. 1981; Suárez-Vázquez, 2011). En la Tabla 1 se muestra la homogeneidad de la muestra con respecto al criterio de selección (frecuencia de acceso a las redes sociales). En ella se puede observar que la mayoría de los participantes acceden a las redes sociales con una frecuencia de entre 7 y 14 veces a la semana.

TABLA 1

\section{FRECUENCIA DE ACCESO A LAS REDES SOCIALES}

\begin{tabular}{|c|c|}
\hline Frecuencia de acceso a las redes sociales & $\%$ \\
\hline Una vez cada 2 semanas & .7 \\
\hline Una vez a la semana & 1.1 \\
\hline Más de una vez a la semana & 8.1 \\
\hline Hasta siete veces a la semana & 37.8 \\
\hline Mínimo 14 veces a la semana & 52.3 \\
\hline Total & 100.0 \\
\hline
\end{tabular}

Fuente: Elaboración propia.

La conveniencia de nuestra muestra también se ve reflejada cuando la comparamos con los segmentos de mayor acceso a las RR.SS. en México. En la Tabla 2 se puede observar que el mayor segmento de usuarios de redes sociales en el país se centra entre las personas de 18 y 34 años, según datos de la Asociación Mexicana de Internet (AMIPCI, 2013)

\section{TABLA 2}

USUARIOS DE REDES SOCIALES EN MÉXICO (EDAD EN PORCENTAJE)

\begin{tabular}{|c|c|}
\hline \multicolumn{1}{|c|}{ Edad de los usuarios de redes sociales } & $\%$ \\
\hline Usuarios de 18 a 24 años & 39 \\
\hline Usuarios de 25 a 34 años & 26 \\
\hline Usuarios de 35 a 44 años & 16 \\
\hline Usuarios de 45 a 54 años & 13 \\
\hline Usuarios de más de 55 años & 6 \\
\hline Total & 100.0 \\
\hline
\end{tabular}

Fuente: AMIPCI (2013).

\section{DISEÑO}

El pre-experimento se llevó a cabo en un entorno natural sin control de las variables del entorno. Si bien, la falta de control sobre las variables exógenas puede ser altamente criticada, hay que considerar que el presente trabajo no pretende medir otros efectos más allá de la emoción o decepción que produzca un consumo y sus efectos sobre la intención de hablar del producto/ servicio.

Como ya se señaló, se empleó el cine como estímulo para analizar el fenómeno boca-oreja en las RR.SS. El uso del cine y, en general, de la industria del entretenimiento como contexto para el análisis de la comunicación boca-oreja virtual es algo común (véase por ejemplo; Godes y Mayzlin, 2004; Liu, 2006; Dellarocas et al. 2007; Duan et al. 2008; Chakravarty et al. 2010). Entre otras cosas, esto se debe a la importancia de la información en el consumo de bienes de experiencia. Para los consumidores, la comunicación boca-oreja resulta una forma efectiva para reducir el riesgo asociado a este tipo de productos. Estudios previos ha identificado que aproximadamente el 50\% de 
los internautas durante sus decisiones de compra de CDs, películas o video juegos, tienden a confiar en las recomendaciones de otros consumidores (Godes y Mayzlin, 2004).

Además, en este trabajo, se empleó a las películas (cine) como estímulo durante este pre-experimento por las similitudes entre los usuarios de las redes sociales virtuales y los espectadores de cine. Como se señaló anteriormente, los mayores usuarios de redes sociales virtuales en México son homogéneos con la muestra analizada (18 a 34 mayores usuarios de RR.SS., frente a un segmento de 18 a 30 años de individuos que participaron en este estudio). En una comparación general de los hábitos de asistencia al cine, se observa que más del 50\% de los participantes declaró asistir en el último trimestre, mientras que tan sólo un 4.6\% señaló hacerlo nunca o casi nunca (véase Tabla 3). De tal modo que, la homogeneidad en ambos segmentos: usuarios de redes sociales y espectadores de cine permite analizar el efecto boca-oreja no sólo entre los mayores usuarios de las RR.SS. sino que también en uno de los productos altamente consumidos por este segmento.

TABLA 3

\section{FRECUENCIA DE ASISTENCIA AL CINE}

\begin{tabular}{|c|c|}
\hline Frecuencia de asistencia al cine & $\%$ \\
\hline Nunca o casi nunca & 4.6 \\
\hline Hace más de un año & 21.6 \\
\hline En el último año & 14.5 \\
\hline En el último trimestre & 59.4 \\
\hline Total & 100.0 \\
\hline
\end{tabular}

Fuente: Elaboración propia.

\section{MEDIDAS}

El efecto boca-oreja se midió a través de una escala de razón que específicamente señalaba el número de personas a las que el participante les diría sobre la película. Esta forma de medida es consistente con la empleada por investigaciones previas [véase por ejemplo a Bowman y Narayandas (2001)].

Para controlar el tipo de película, a través de una variable dicotómica (cualitativa nominal) se codificaron los dos posibles escenarios ( 1 = película emocionante y 2 = película decepcionante).

\section{RESULTADOS}

Este trabajo se concentró en analizar los efectos de las experiencias tanto emocionantes como decepcionantes sobre la comunicación boca oreja en las RR.SS. Para lograrlo, se empleó la prueba t que nos permite evaluar la diferencia entre dos medias. Concretamente, se empleó la prueba t para muestras relacionadas que es la más apropiada cuando, como en nuestro caso, el experimento cuenta con dos condiciones (películas emocionantes y películas decepcionantes) en las que el mismo grupo de participantes toma parte.

El test realizado en este pre-experimento ofrece resultados congruentes con la literatura previa (véase por ejemplo: Mazzarol et al. 2007), reflejando diferencias significativas entre la emoción o decepción que pueda provocar una película y el número de personas con las que comentarían sobre ella: $\mathrm{t}(280)=-4.57, \mathrm{p}=.000$ (véase Tabla 4).

Como se esperaba, el volumen de boca oreja virtual cambia dependiendo de la experiencia vivida. Sin embargo, aún no se aclara cual experiencia desencadena mayor comunicación. Los estadísticos descriptivos confirman la intuición señalada en el presente trabajo. Así pues, cuando los individuos se enfrentan a una experiencia negativa (películas decepcionantes), los participantes tienden a hablar más de la película (en 
promedio comunican su experiencia hasta con 8 personas dentro de su red social). Mientras que cuando éstos se enfrentan a una experiencia positiva (películas emocionantes) tienden a hablar menos (en promedio hablan de la película con 5 personas dentro de su red social). Los resultados sugieren que, cuando las personas se enfrentan a una experiencia desagradable, la propagación del boca oreja virtual, incrementa en dos personas más comparado a la propagación que se suscita cuando se enfrentan a experiencias agradables (véase Tabla 5).

\section{DISCUSIÓN Y CONCLUSIONES}

En el presente trabajo, a través de un pre-experimento y utilizando una Prueba t para muestras relacionadas, se analizaron las experiencias emocionantes y decepcionantes ocasionadas por las películas y sus efectos sobre la cantidad (volumen) de comunicación boca-oreja que estas experiencias generan en las redes sociales. Nuestros resultados muestran que el bocaoreja en las redes sociales tiene más volumen (se genera en mayor cantidad de e-WOM) cuando los individuos se enfrentan a experiencias decepcionantes.
La importancia de nuestros resultados aumenta al enfrentarlos con la evidencia previa que señala que el volumen de boca-oreja, es decir la cantidad de cometarios entre consumidores, afecta sensiblemente la decisión de compra de los individuos (Anderson y Salisbury, 2003; Bowman y Narayandas 2001; Van den Bulte y Lilien 2001).

En el caso de los bienes de experiencia (y en concreto el cine y la televisión), las investigaciones sugieren que mayores niveles de bocaoreja están relacionados con mayores ventas y recaudación de taquilla, esto se debe al poder informativo del boca-oreja. Por lo tanto, cuando aumenta la comunicación entre consumidores sobre un producto o servicio, aumenta el conocimiento que se tiene sobre éste y, por lo tanto, la probabilidad de que se venda (Godes y Mayzlin 2004; Liu 2006). Como ya se señaló, los efectos del boca-oreja negativo son mayores que los del positivo. Típicamente, se considera que, mientras la comunicación positiva entre consumidores alienta el consumo de un determinado bien o servicio e incrementa las expectativas de calidad, la comunicación boca-oreja negativa desalienta el consumo y disminuye las expectativas.

TABLA 4

PRUEBA T DE MUESTRAS RELACIONADAS

\begin{tabular}{|c|c|c|c|c|}
\hline & Media & Desviación Estándar & $t$ & Significatividad \\
\hline Experiencia emociónate -Experiencia decepcionante & -2.18 & 7.98 & -4.57 & 0.000 \\
\hline
\end{tabular}

Fuente: Elaboración propia.

TABLA 5

TABLA 5. ESTADÍSTICOS DESCRIPTIVOS POR GRUPOS

\begin{tabular}{|c|c|c|c|}
\hline \multicolumn{2}{|c|}{ Media } & Desviación Estándar & N \\
\hline Experiencia emocionante & 5.88 & 7.64 & 281 \\
\hline Experiencia Decepcionante & 8.06 & 10.55 & 281 \\
\hline Diferencia Media & -2.18 & & \\
\hline Fuente: Elaboración propia.
\end{tabular}

Fuente: Elaboración propia. 
Así, esta investigación sugiere que las experiencias decepcionantes conducirán a más comunicación boca-oreja negativa. De confirmarse este supuesto, estaríamos frente a un problema de gestión en el caul una decepción no sólo conduciría a un mayor volumen de boca-oreja sino que, probablemente esté propiciando comunicación negativa y, por tanto, desalentando el consumo de un producto o servicio en particular.

Estos resultados, son de especial interés para los ejecutivos de la industria del cine pues la comunicación boca-oreja es especialmente influyente durante el fin de semana de estreno de las películas, donde el boca-oreja positivo puede ayudar a una película a permanecer más tiempo en cartelera. Mientras que el boca-oreja negativo puede hacer que una película permanezca en cartelera menos tiempo del que estaba planeado (Eliashberg et al. 2006).

\section{FUTURAS LÍNEAS DE INVESTIGACIÓN}

En el presente trabajo se advirtió que quienes sufren experiencias decepcionantes son quienes inician un proceso de comunicación boca-oreja con mayor intensidad (volumen). Sin embargo, no se analizó el sentido de esa información, aunque la intuición es que comparten una opinión negativa. Futuras investigaciones pueden concentrarse en qué tipo de comunicación generan las diferentes experiencias (emocionantes y decepcionantes) y determinar si, efectivamente, las experiencias decepcionantes conducen a una comunicación negativa entre los consumidores.

Por otra parte, aunque se advirtió que las experiencias decepcionantes, frente a las emocionantes, generan más boca-oreja (aproximadamente, una experiencia decepcionante se comunica 2.5 veces más que una emocionantes), no se analizó qué información, ya sea la procedente de experiencias emocionantes o la de experiencias decepcionantes, es la que tiene mayor influencia en las decisiones de los consumidores. Futuras investigaciones podrían concentrarse en los efectos de ambos tipos de información sobre la decisión de ver una película.

\section{REFERENCIAS}

AMIPCI. (2013). MKT digital y redes sociales en México 2013. Recuperado de: http://www.amipci.org. $\mathrm{m} \times / ? \mathrm{P}=$ edito multimediafleyMultimedia $=435 \mathrm{y} T$ ype $=1$

Anderson, E. and Salisbury, L. (2003). The formation of market-level expectations and its covariates, Journal of Consumer Research, 30, 115-124.

Arenas-Gaitan, J., Rondan-Cataluña, F. J., and RamírezCorrea, P. E. (2013). Social identity, electronic word-of-mouth and referrals in social network services. Kybernetes, 42, 1149-1165.

Bansal, H.S. and Voyer, P.A. (2000). Word-of-mouth processes within a services purchase decision context. Journal of Service Research, 3, 166-77.

Bowman, D. and Narayandas, D. (2001). Managing customer-initiated contacts with manufacturers: The impact on share of category requirements and word-of-mouth behavior. Journal of Marketing Research, 38, 291-297.

Calder, B.J., Phillips, L.W. and Tybout, A.M. (1981). Designing research for application. Journal of Consumer Research, 8, 197-207.

Chakravarty, A., Liu, Y. and Mazumdar, T. (2010). The Differential Effects of Online Word-of-Mouth and Critics Reviews on Pre-release Movie Evaluation.

Casaló, L. V., Flavián, C. and Guinalíu, M. (2008). The role of satisfaction and website usability in developing customer loyalty and positive word-ofmouth in the e-banking services. International. Journal of Bank Marketing, 26, 399-417.

Chakravarty, A., Liu, Y., and Mazumdar, T. (2010). The differential effects of online Word-of-Mouth and critics' reviews on pre-release movie evaluation. Journal of Interactive Marketing, 24, 185-197.

Chung, C. M., and Tsai, Q. (2009). The effects of regulatory focus and tie strength on word-of-mouth 
behaviour. Asia Pacific Journal of Marketing and Logistics, 21, 329-341.

Dellarocas, C., Zhang, X. and Awad, N. (2007). Exploring the Value of Online Product Reviews in Forecasting Sales: The Case of Motion Pictures. Journal of Interactive Marketing, 21(4), 23-45.

Duan, W., Gu, B. and Whinston, A. (2008). The Dynamics of Online Word-of-Mouthand Product Sales-An Empirical Investigation of the Movie Industry. Journal of Retailing, 84(6), 233-242.

Eliashberg, J., Jonker, J. J., Sawhney, M. S. and Wierenga, B. (2000). MOVIEMOD: An implementable decision-support system for prerelease market evaluation of motion pictures. Marketing Science, $19,226-243$.

Engel, J.F., Kegerreis, R. and Blackwell, R. (1969). Wordof-mouth communication by the innovator. Journal of Marketing, 33, 15-19.

Gilly M., Graham J., Wolfinbarger M. and Yale L. (1998). A dyadic study of interpersonal information search. Academy of Marketing Science Journal, 26, 83-100.

Godes, D. and Mayzlin, D. (2004). Using Online Conversations to Study Word-of-Mouth Communication. Marketing Science, 23(4), 545-560.

Granovetter, M.S. (1973). The strength of weak ties. American Journal of Sociology, 78, 1360-1380.

Hair, J. F. (2006). Análisis multivariante. Madrid: Prentice Hall.

Herr, P., Kardes, F. \& Kim, J. (1991). Effects of word-ofmouth and product attribute information on persuasion: An accessibility-diagnosticity perspective. Journal of Consumer Research, 17, 454-462.

Jedidi, K., Krider, R. and Weinberg, Ch. (1998). Clustering at the movies. Marketing Letters, 9(4), 393-405.

Katz, E. and Lazarsfeld, P. (1955). Personal Influence. Free Press: Glencoe, IL.

Kyung Kyu K. and Prabhakar B. (2004). Initial Trust and the Adoption of B2C e-Commerce: The Case of Internet Banking. Database for Advances in Information Systems, 35, 50-64.
Liu, Y. (2006). Wor-of-Mouth for Movies: Its Dynamics and Impact on Box Office Revenue. Journal of Marketing, 70(3), 74-89.

Mazzarol, T., Sweeney, J. C., and Soutar, G. N. (2007). Conceptualizing word-of-mouth activity, triggers and conditions: an exploratory study. European Journal of Marketing, 41, 1475-1494.

Reinstein, D.A., \& Snyder, C.M. (2005). The influence of expert reviews on consumer demand for experience goods: A case study of movie critics. The Journal of Industrial Economics, 53(1), 27-51.

Richins, M. (1983). Negative word-of-mouth by dissatisfied consumers: a pilot study. Journal of Marketing, 47, 68-78.

Sen, S., and Lerman, D. (2007). Why are you telling me this? An examination into negative consumer reviews on the Web? Journal of Interactive Marketing, 21(4), 76-94.

Suárez-Vázquez, A. (2011). Critic power or star power? The influence of hallmarks of quality of motion pictures: an experimental approach. Journal of Cultural Economics, 35, 119-135.

Tran, G., Strutton, D., and Taylor, D. (2012). Do microblog postings influence consumer perceptions of retailers'e-servicescapes? Management Research Review, 35, 818-836.

Van den Bulte, C. and Lilien G. (2001). Two-stage partial observability models for innovation adoption, working paper, Wharton School, University of Pennsylvania.

Yang, J., Kim, W., Amblee, N., and Jeong, J. (2012). The heterogeneous effect of WOM on product sales: why the effect of WOM valence is mixed? European Journal of Marketing, 46, 1523-1538.

Yongseog K. (2005). The effects of buyer and product traits with seller reputation on price premium. The Journal of Computer Information Systems, 46, 79-91.

Recibido: 29 de mayo de 2015 Aceptado: 10 de diciembre de 2015 
This document was created with Win2PDF available at http://www.win2pdf.com. The unregistered version of Win2PDF is for evaluation or non-commercial use only. This page will not be added after purchasing Win2PDF. 